\title{
Relativistic Jets in Active Galactic Nuclei and their Relationship to the Central Engine
}

\author{
Alan P. Marscher \\ Institute for Astrophysical Research, Boston University \\ 725 Commonwealth Ave., Boston, MA 02215 USA \\ E-mail: marscher@bu.edu
}

The jets in microquasars have a number of properties in common with relativistic jets in radioloud active galactic nuclei (AGN). Blazar jets are easier to observe, which allows us to follow changes in the structure, continuum spectrum, and polarization and to use these variations to survey the jets and deduce their physical properties. I review some basic characteristics of blazar jets in order to facilitate comparison with X-ray binary systems (XRBs). The Lorentz factor of the flow can be as high as $\sim 45$, but in most objects is less than 5 . An observed inverse relationship between Lorentz factor and intrinsic opening angle can be explained if the flow accelerates and collimates over an extended region. Superluminal knots are well explained by shock waves propagating down the jet. The magnetic field is turbulent downstream of the "true" core, which probably coincides with the end of the acceleration/collimation zone. The magnetic field in the latter should have a well-ordered tight helical geometry, but this may only be apparent at short $\mathrm{mm}$ and submm wavelengths I report on X-ray and radio timing observations of two blazar-like radio galaxies, 3C 120 (Fanaroff-Riley type 1) and 3C 111 (FR 2). In both objects, minima in the X-ray light curves precede the appearance of bright knots moving down the radio jet as seen in sequences of Very Long Baseline Array (VLBA) images. While not as dramatic as the state changes in the X-ray emission from microquasars, the radio galaxy observations demonstrate that, as in XRBs, there is an intimate connection between X-ray production in the corona (which may be the base of the jet) and energy injected into the jet flow.

VI Microquasar Workshop: Microquasars and Beyond Societa' del Casino, Como, Italy

18-22 September, 2006 


\section{Introduction}

The discovery [32] that X-ray binary (XRB) systems possess relativistic jets has led to a quantum leap in our ability to explore the mechanisms by which accreting black holes and neutron stars produce highly collimated outflows with velocities close to the speed of light $c$. The distinct X-ray states and the well-defined differences in jet flow properties demonstrate that the jet is intimately connected with the central engine.

Microquasars are ideal for studying the central engine, but it is not so easy to gather all the data needed to define the physical properties of the jets. This is because of moderate flux levels and variability so rapid that the jet structure varies during the time that the Earth's rotation allows an array to sample the full range of interferometric $u, v$ spatial frequencies needed to make a high dynamic-range image. In AGN, the central engines of Seyfert galaxies generate the $\mathrm{X}$-ray emission but the jets tend to be weak and not particularly relativistic. In blazars, emission from the jets dominates essentially the entire electromagnetic spectrum, so we can probe the jets using observations of the time-variable images (with VLBI), flux, and polarization. This proves to be a complex enterprise, since we need to observe a blazar regularly over many frequencies and then take Doppler beaming and other relativistic effects into account when interpreting the observations.

Fortunately, there are a few radio galaxies that combine the advantages of Seyferts and blazars. These have jet-dominated emission at $\mathrm{cm}$ and $\mathrm{mm}$ wavelengths while the uv and X-ray emission is generated mainly by the central engine. Along with my collaborators, I have been monitoring two such radio galaxies, 3C 120 and 3C 111. Here I report on what we have learned thus far about these AGN. First, however, I summarize the properties of relativistic AGN jets inferred from observations of the more extreme blazars.

\section{Physical Characteristics of Blazar Jets}

\subsection{Kinematic Parameters}

The derivation of the physical parameters of a relativistic jet requires knowledge of the effects of relativistic beaming and light-travel delays. That is, we need to know the Doppler beaming factor $\delta$, Lorentz factor $\Gamma$, and angle between the velocity vector and the line of sight $\theta$. The synchrotron flux density is amplified by $\delta^{3+\alpha}\left(\delta^{2+\alpha}\right.$ if the emission is in a stationary feature through which the jet plasma flows), where $-\alpha$ is the slope of the log flux density vs. $\log$ frequency spectrum. Furthermore, a feature (e.g., a shock front) moving down the jet passes a distance $\Gamma \delta \beta c t$ over observer's time $t$, where $\beta$ is the speed of the feature divided by the speed of light. Observed frequencies are a factor $\delta$ higher than those emitted in the proper frame of the emitting plasma. The actual opening angle of the jet is $\tan \theta$ times smaller than the observed value projected on the sky.

Until recently, we could not determine accurately the values of $\Gamma, \delta$, and $\theta$ separately. The standard method (used, e.g., by [20], who discuss its inaccuracy) has been to measure the 
apparent speed of a bright knot, $\beta_{\text {app }}=\frac{\beta \sin \theta}{1-\beta \cos \theta}$, from sequences of VLBI images, and to assume that the viewing angle $\theta$ is the value that maximizes $\beta_{\text {app }}, \theta=\sin ^{-1}\left(\Gamma^{-1}\right)$. In this case, $\delta=$ $\Gamma=\beta_{\text {app. }}$. But population statistics of relativistically beamed sources (e.g., [24]) indicate that the flux limited samples from which most radio-loud AGN have been selected favor smaller viewing angles such that $\delta>\Gamma$ and $\delta>\beta_{\text {app }}$. [23] estimate the value of $\delta$ independently of $\beta_{\text {app }}$ by first determining the "variability brightness temperature" $T_{\text {var }}$ under the assumption that the source size equals the speed of light multiplied by the timescale of variability of the 37 or 22 $\mathrm{GHz}$ light curve. The Doppler factor required to bring $T_{\text {var }}$ down to some theoretical value in the source frame, $T^{\prime}$, is then calculated and adopted. The problem with this method is that there are at least two values of $T^{\prime}$ that one might adopt. The first is very rough, $\sim 10^{12} \mathrm{~K}$, above which the luminosity from inverse Compton emission exceeds that from synchrotron radiation [21]. The second, which is better specified - several times $10^{10} \mathrm{~K}$ - corresponds to equipartition of the energy density in magnetic field with that in relativistic electrons [35]. However, [10] have found that superluminal knots tend to have brightness temperatures that significantly exceed the equipartition value, suggesting that the knots are probably shock waves within which much of the energy goes into particle acceleration.

[16] have developed a technique for determining $\Gamma, \delta$, and $\theta$ that is more precise and not so strongly dependent on assumptions. The method is based on the measurement of the rate of decline of the flux density of an individual knot as measured in sequences of $43 \mathrm{GHz}$ VLBA images (see Fig. 1). If the flux decrease is modulated by light-travel delays across the emission region, then the timescale of flux decrease, $t_{\mathrm{var}}$, is related to the size of the region and the Doppler factor is given by $\delta=\frac{a D}{c t_{\mathrm{var}}(1+z)}$, where $a$ is the angular diameter as measured from model fitting the VLBI data, $D$ is the luminosity distance, and $z$ is the redshift. Use of this equation requires that the radiative energy losses of the relativistic electrons dominate over expansion cooling. This is generally true at $43 \mathrm{GHz}$ : [16] found that the decrease in flux density is substantially faster than would be the case if cooling from the observed expansion of a knot were the cause. (This makes sense: the intrinsic opening angles of the jets are very small, so lateral expansion occurs only gradually as a knot propagates downstream.) At lower frequencies expansion cooling probably dominates in many cases (see, e.g., [12]). The extent of a knot along the line of sight direction - which determines the light-crossing time delay - is assumed to be about the same as the transverse size measured from the VLBI data. This might not work if a knot is thin, as in the shock model of [26] at high frequencies owing to strong energy losses of the highest energy electrons, and observed transversely to its face. However, at $43 \mathrm{GHz}$ the radiative zone is likely to be only a bit thinner than the full extent of the shocked region since the electrons have intermediate energies and thus can travel fairly far from the shock front before radiating most of their energy. Furthermore, lines of sight directly transverse to a shock front occur with low probability. For other line-of-sight directions, relativistic aberration rotates the shock so that it is seen at a large angle - edge-on if $\theta \approx \sin ^{-1}\left(\Gamma^{-1}\right)-$ and the line-of-sight dimension is about the same as the transverse extent. 


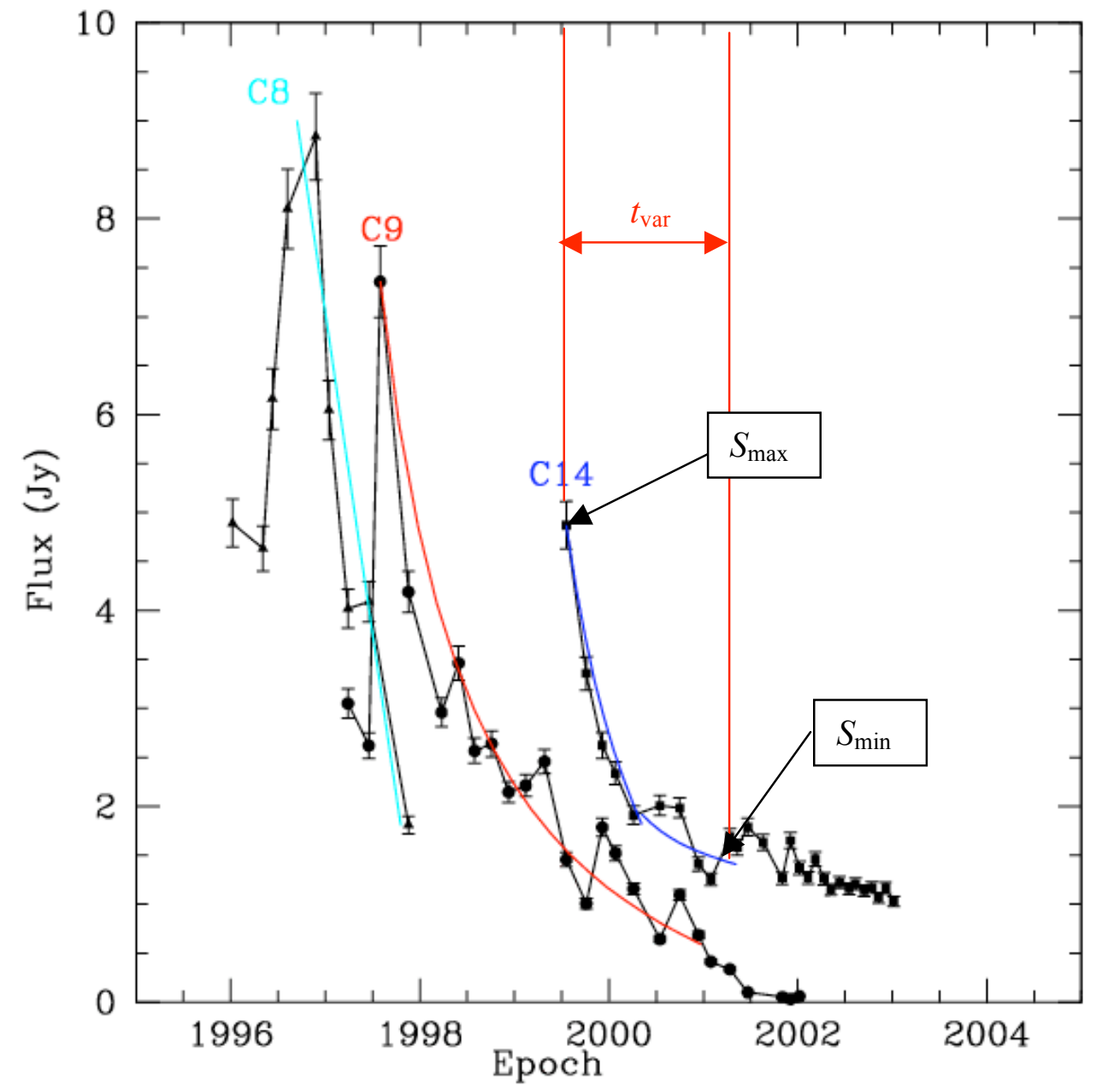

Figure 1: Measurement of the timescale $t_{\mathrm{var}}$ of the decrease in flux density of a superluminal knot observed in sequences of VLBA images of the quasar 3C 279 (data from [16]). The flux densities are derived from modeling the jet by multiple discrete components and comparing the visibilities of the model with the actual VLBA interferometric visibility data. The VLBA data are corrected for antenna gain variations, sky opacity, and atmospheric phase delays.

Although the bulk motion of the emitting plasma may not be the same as the motion of the centroid of the knot as measured on the VLBI images, if the knot is a shock wave the difference is not large. If the plasma has an ultrarelativistic equation of state, the velocity of the plasma relative to the shock front is $c / 3$. In this case the Lorentz factor of the plasma in the observer's frame is $\Gamma_{\text {plasma }}=1.06 \Gamma_{\text {shock }}\left(1-\beta_{\text {shock }} / 3\right)$. For example, if $\Gamma_{\text {shock }}=20$ then $\Gamma_{\text {plasma }} \approx 14$ and the centroid of the knot moves at a Lorentz factor between these two values, about $17 . \Gamma_{\text {plasma }}$ is even closer to $\Gamma_{\text {shock }}$ if the protons are not highly relativistic, in which case the plasma velocity relative to the shock front $<c / 3$. Thus, the approximation that $\Gamma_{\text {plasma }} \approx \Gamma_{\text {centroid }}$ is accurate for extreme blazars with $\Gamma>10$ that are the subjects of most in-depth studies.

The derived Doppler factor gives one relation between $\Gamma$ and $\theta, \delta=[\Gamma(1-\beta \cos \theta)]^{-1}$, where $\beta=\sqrt{1-\Gamma^{-2}}$. Measurement of the apparent speed $\beta_{\text {app }}$ in the same knot gives a second relation 
(see formula a few paragraphs above). Together, the two equations provide independent values of $\Gamma$ and $\theta$, thus determining the kinematic parameters of the jet.

[16] have applied this method to 15 AGN observed $\sim$ bi-monthly over a three-year period. The derived Doppler factors of some extreme blazars (e.g., 3C 279; [18]) are much higher than previously inferred from apparent velocities alone, which is comforting. The highest measured robustly (using polarization to identify the knots on time sequences of images) $\sim 45 \mathrm{c}$ in the quasar PKS 1510-089 (see Fig. 2). Comparison of Doppler factors derived from the above method with minimum values of $\Gamma$ needed to explain the superluminal apparent speed, $\Gamma_{\min }=\sqrt{1+\beta_{\text {app }}^{2}}$, demonstrates that the latter are $0.72 \pm 0.15$ times $\Gamma_{\min }$, as expected according to population statistics of relativistically beamed sources [24] to within the uncertainties. The method therefore appears to be quite robust, an indication that the approximations and assumptions lead to only minor differences between the derived and actual values of $\Gamma$.

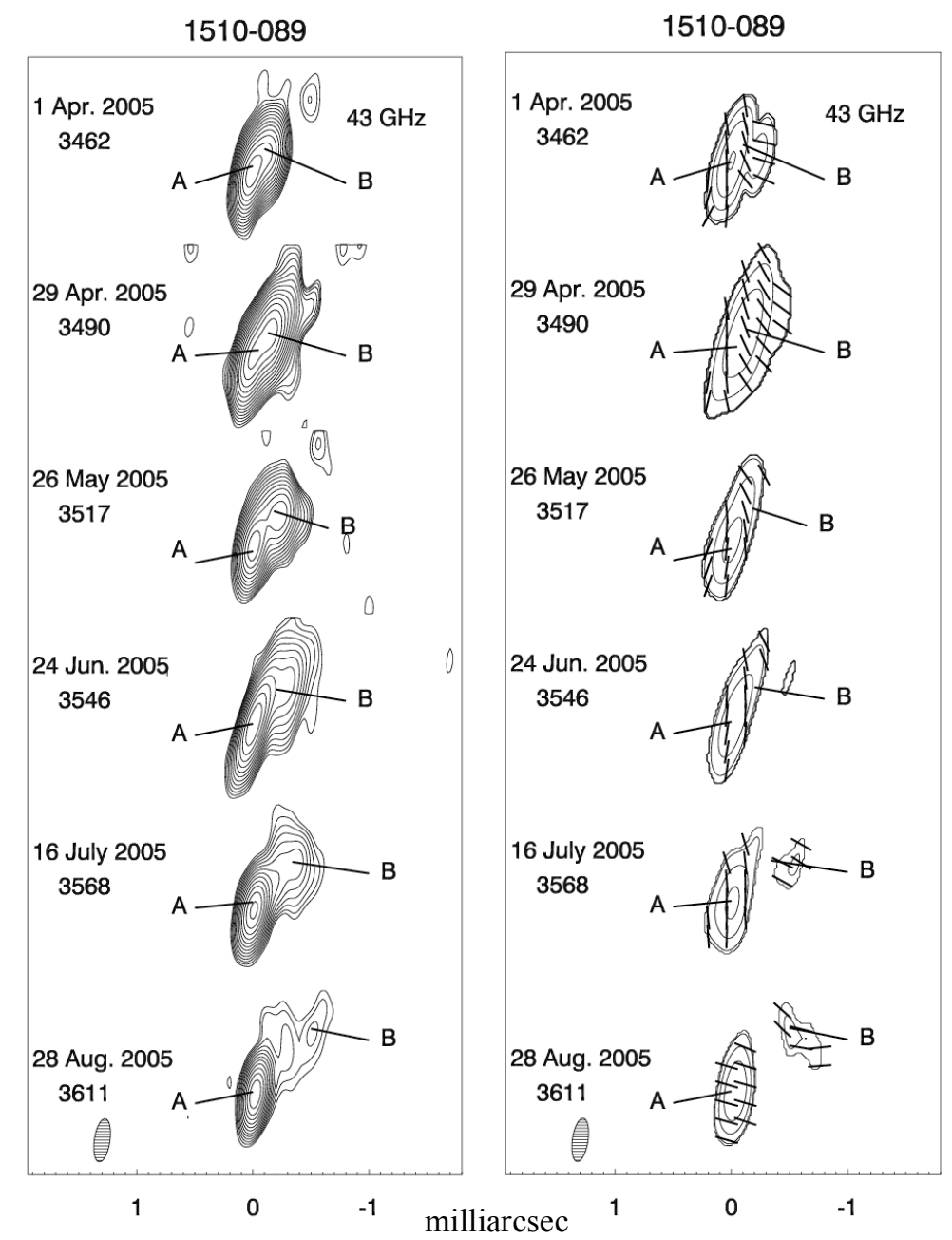

Figure 2. Total intensity (left) and polarized intensity (right; lengths of sticks indicate fractional polarization and orientations correspond to the electric field vector directions) images of the quasar PKS 1510-089. Knot $B$, identified by its polarization angle, moves away from the core $(A)$ at $\sim 2$ milliarcsec $\mathrm{yr}^{-1}$, or about $45 c$ for a Hubble constant of $71 \mathrm{~km} \mathrm{~s}^{-1} \mathrm{Mpc}^{-1}$. 
Application of the method to $3 \mathrm{C} 273$, whose jet is often rather broad as projected on the sky, yields a higher Lorentz factor $(\Gamma \approx 13)$ for knots that travel along a southern route that is different for the northern features $(\Gamma \approx 8)$. In fact, in 2002-2005 a pair of knots appeared alongside each other across the width of the jet, each moving with a different apparent speed [37]. Velocity shear represented by this cross-jet gradient can explain why there is a modest ordered component to the magnetic field parallel to the jet in this and many other quasars [36].

\subsection{Population Statistics of Jet Lorentz Factors}

The fastest AGN jets are found in $\gamma$-ray bright blazars ([19],[20],[34]). In a VLBA survey of about $60 \%$ of the EGRET-detected sources identified as blazars, [19] reported apparent velocities as high as $\sim 45 c$ in PKS 1510-089, confirmed by [16] and by the data shown in Figure 2. But a typical radio-loud AGN is not so fast. Apparent velocities in radio galaxy jets with wide viewing angles are typically $0.2-0.8 c$. Our impression of typical Lorentz factors is influenced by the high- $\Gamma$ blazars that overpopulate any centimeter-wave survey of bright, flatspectrum sources because of beaming bias. A volume-limited sample of radio-loud AGN would be dominated by objects with more mundane jets. In a population synthesis study, [24] used realistic luminosity functions to show that the majority of compact extragalactic radio sources have jets with low Lorentz factors. If the Lorentz factor distribution of the population is a power law, $N(\Gamma) \propto \Gamma^{-a}$, a value of $a$ between 1.5 and 1.75 provides a good match between the synthetic and observed distribution of apparent velocities. If the Lorentz factors of the population ranges from $\Gamma_{\min } \sim 1.2$ to $\Gamma_{\max } \sim 45$, then the allowed range for $a$ means that between 83 and $88 \%$ of compact jets have Lorentz factors $<10$ and $44-47 \%$ have $\Gamma<3$.

\subsection{Jet Opening Angles}

Given the knowledge of the viewing angle of the jet (i.e., $\theta$ for knots moving parallel to the axis), we can determine the actual opening angle $\psi$. [16] have done this for the 15 AGN they studied, finding that the opening angle is inversely proportional to the flow Lorentz factor. This makes sense if the jet is accelerated and collimated over an extended region [38], which probably corresponds to the span between the central engine and the core of the jet at the frequency where the self-absorption spectral turnover occurs, $\sim 1$ to $3 \mathrm{~mm}$ ([14],[1]). Since the jet becomes ever more collimated as $\Gamma$ increases, the extended acceleration picture approximately reproduces the relation $\psi \propto \Gamma^{-1}$. This explains why the apparent opening angles, which are roughly the true opening angle $\psi$ divided by $\tan \theta$, do not correlate with $\Gamma$, since both $\psi$ and $\tan \theta$ are inversely proportional to $\Gamma$.

\subsection{Shock Waves as the Paradigm for Moving Knots and the Radio Core}

The most popular model for the superluminal knots in AGN involves shock waves propagating down the jet (e.g., [26],[12]). The linear polarization has the characteristics of a a shock wave compressing a turbulent ambient magnetic field ([11],[17]). This is indicated by the relatively low fractional polarization. If we approximate the turbulence as $N$ cells of independent, randomly oriented magnetic fields, the polarization without 
compression $p \approx 0.7 / \sqrt{N}$ and it fluctuates at a level of $\sigma(p) \approx 0.7 / \sqrt{2 N} \approx p / \sqrt{2}$ [2]. The compression increases $p$ and reduces $\sigma(p)$ by an amount that depends on the level of compression and angle between the (aberrated) line of sight and shock plane [13]. [11] points out that the compression needed is small for most knots - typically $\sim 20 \%$ - so that the shocks are fairly weak.

What appears as the core on VLBI images is either the point where the optical depth becomes of order unity at the wavelength of observation or a standing shock. Probably it is the former in some AGN and the latter in others. Evidence for shocks comes from seeing stationary features downstream of the core in many compact jets $([19],[16])$ - which indicates that standing shocks are common - and from the polarization in some cores at $43 \mathrm{GHz}$ matching the expectations of standing conical shocks ([3],[4],[7]).

\subsection{The Acceleration/Collimation Zone}

The picture of a jet containing a turbulent magnetic field contrasts with the ordered field geometry needed for acceleration and collimation in the prevailing magnetic launching models (e.g., [30],[38],[29],[9]). [17] find suggestions that the polarization at $1 \mathrm{~mm}$, where the jets are optically thin even in the "true" core, is relatively high and distinct from the longer mm-wave and optical polarization. One interpretation is that an emission component from the acceleration/collimation zone is present at $1 \mathrm{~mm}$. This implies that there is a rather abrupt transition between the ordered field - a tight helix according to the standard theories - and the turbulence (see Fig. 3).

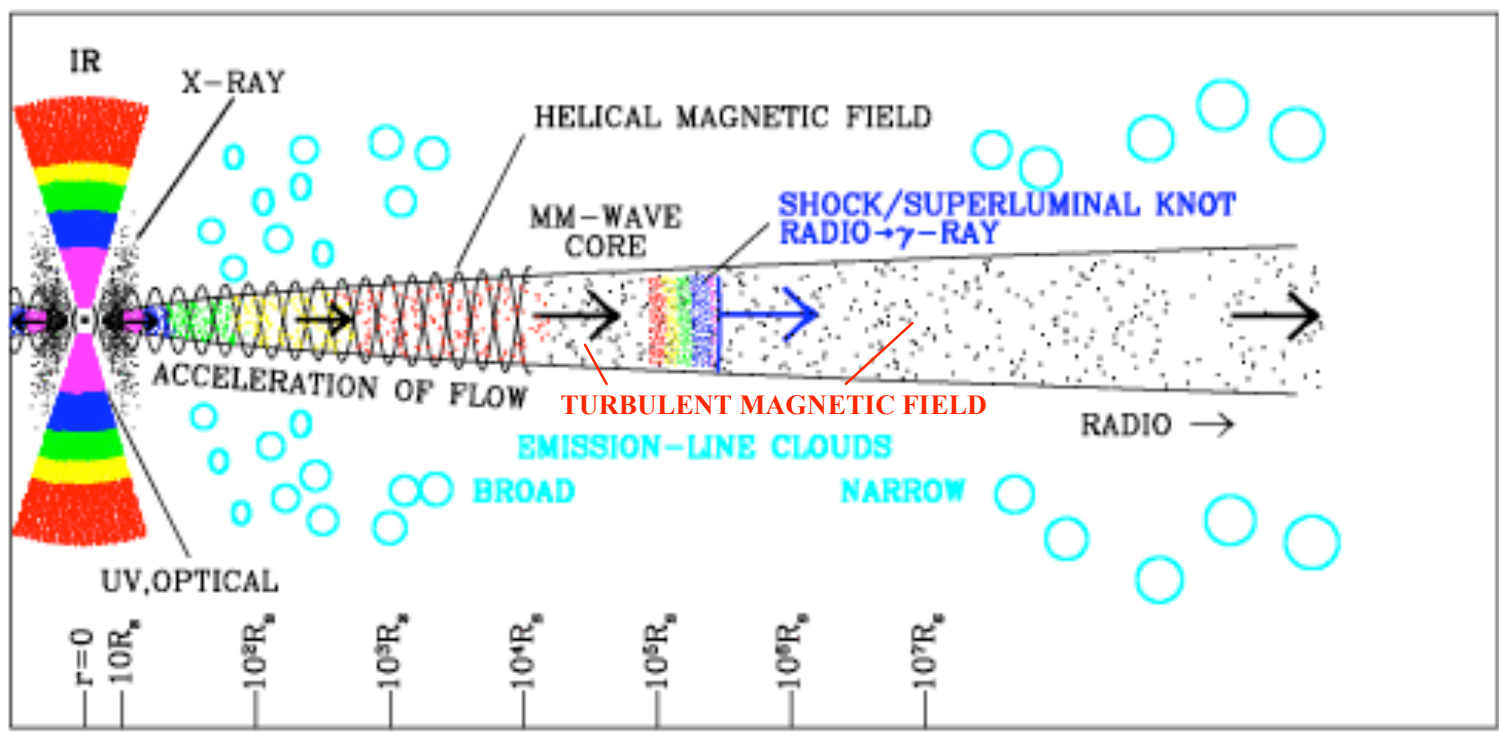

Figure 3. Sketch of main features of an AGN according to observations and prevailing models. Note the logarithmic length scale, marked on the bottom. (The sketch is cut off on the left side, which is roughly symmetric with the right side.) Black hole is the dot at the center of the flared disk to the left. Shock waves are frequency-stratified, with highest synchrotron frequencies emitted only close to the shock front where electrons are energized [26]. The part of the jet interior to the mm-wave core is opaque at $\mathrm{cm}$ wavelengths. At this point, it is not clear whether substantial emission occurs between the base of the jet and the mm-wave core. 
The brightest spot of the ambient jet is the core when observed at or above the turnover frequency of the overall flux density vs. frequency spectrum. If $N_{0}$ is the normalization of the electron energy distribution and $B$ is the magnetic field at a position where the width of the jet is $R$, the flux per unit jet length is roughly proportional to $N_{0} B^{1+\alpha} \delta^{2+\alpha} R^{2}$. In the conical part of the jet beyond the acceleration/collimation zone, this is a decreasing function of distance down the jet. In the acceleration zone, however, it can increase with distance down the jet as $\delta$ and $R$ increase if this more than compensates for the drop in $N_{0}$ and $B$. Then the flux per unit length reaches a maximum near the point of transition between the acceleration/collimation and ballistic/conical zones. For a jet viewed at an angle $\gg \Gamma^{-1}$, however, as in many radio galaxies, the Doppler factor instead decreases as $\Gamma$ increases, so the brightest spot in the jet is as far upstream as there exist electrons with energies high enough to radiate at the frequency of observation. VLBI observations of side-on radio-galaxy jets at $\mathrm{mm}$ wavelengths can therefore probe the collimation of the jet and its ability to accelerate particles as a function of distance from the central engine, while similar observations of blazar jets can probe the jet's acceleration to a high flow Lorentz factor.

The asymptotic Lorentz factor in the magnetic launching models is roughly half the ratio of the magnetic to rest-mass energy density at the base of the jet, $\Gamma \approx \frac{B^{2}}{16 \pi \rho c^{2}}$. This implies that a change in the mass loading into the jet at its launching pad in the central engine can result in the Lorentz factor downstream taking on a different value. If the mass input were to decrease, the ultimate Lorentz factor of the jet should increase, with the core shifting downstream if the acceleration is distributed, as in the Vlahakis \& Konigl [38] model. This would drive a shock wave into the jet, with the shock forming only at the end of the acceleration zone. (I first heard this idea from G. Ghisellini.)

\section{Connection between the Central Engine and Jet in Radio Galaxies}

Along with my collaborators, I have been observing the radio galaxies 3C 120 (FR 1) and 3C 111 (FR 2) in an attempt to determine whether there is a link between events in the X-ray light curves and in the jet, as in XRBs. These radio galaxies have X-ray emission mostly from the central engine [5] as well as relativistic jets featuring apparent superluminal motion ([8],[16]). Monitoring of 3C 120 with RXTE and the VLBA from early 1997 to early 2000 revealed that superluminal "ejections" follow marked dips in X-ray flux accompanied by hardening of the X-ray spectrum [28]. There is a time delay of 60 days (updated value) that is partly caused by the offset of the core from the central engine, which is more than $0.4 \mathrm{pc}$ or 160,000 gravitational radii for a black-hole mass of $5 \times 10^{7} \mathrm{M}_{\text {sun }}$ [33], and partly by the core shift between the observation frequency of $43 \mathrm{GHz}$ and the self-absorption turnover frequency $v_{\mathrm{m}} \approx$ $100 \mathrm{GHz}$ [1]. More intense monitoring over the past four years (Fig. 4) confirms this trend, with the quantitative addition of a formal, although modest, anticorrelation between X-ray flux and high-frequency radio flux. 


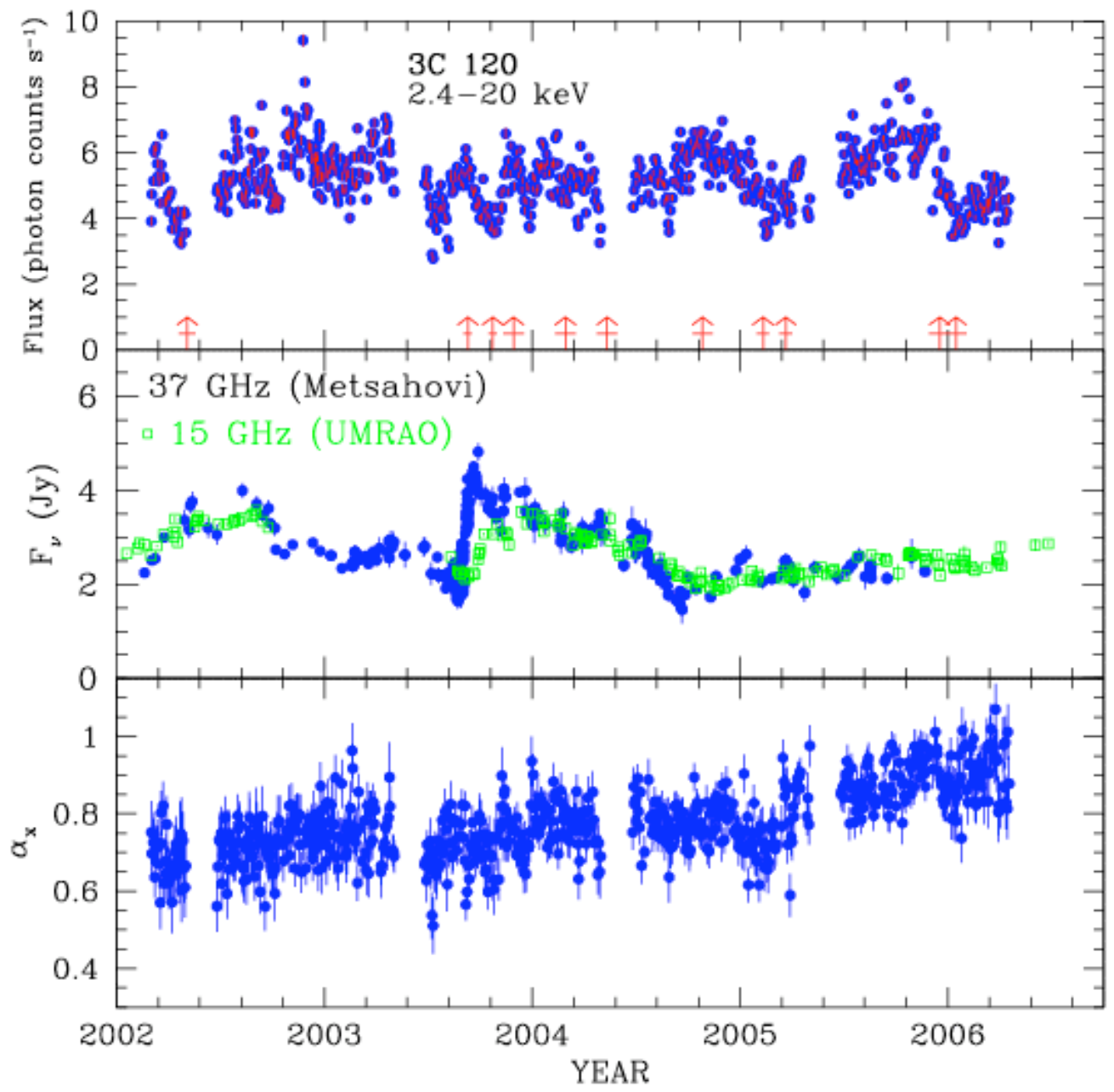

Figure 4. X-ray and radio light curves of the FR 1 radio galaxy 3C 120 since 2002. Upward red arrows indicate times when superluminal knots were coincident with the core on $43 \mathrm{GHz}$ VLBA images (horizontal bars across the arrows show the uncertainty in this time). Every significant dip in X-ray flux and hardening of the X-ray spectrum precedes the ejection of a bright superluminal knot.

The X-ray dips in 3C 120 are not nearly as dramatic as are the transitions between X-ray states in GRS 1915+105, but they are not minor - typically of order a factor of 2 decrease in flux. If the time scales in 3C 120 were shortened by a factor of 3 million according to the ratio of the black-hole masses, the dips would last for $\sim 1 \mathrm{~s}$. There are in fact variations of about this amplitude and time scale in GRS 1915+105 [6], but no connection of these to the energy flow in the jet is apparent. Perhaps we are too demanding to expect direct scaling over so many orders of magnitude in size scale. Nevertheless, we can declare that the radiative state of the accretion disk plus corona system has a direct affect on events in the jet of this AGN, as it does in XRBs.

We have been monitoring the FR 2 radio galaxy 3C 111 only since March 2004. As Figure 5 shows, the results thus far strongly suggest a similar relation between low X-ray states and the production of a bright superluminal knot in the jet. The first and third knots, whose appearances are marked by upward arrows in Figure 5, were initially brighter than the core. Of particular note is the prolonged low-hard X-ray state in 2005. The superluminal knot that 
emerged a few months after the first minimum/hardening during this state was trailed by a string of bright emission (see Fig. 6), commensurate with the extended duration of the low X-ray state. Despite its rather unspectacular appearance in the light curve, the spectrally hard drop in X-ray flux in early 2005 was about a factor of 2 lower than the maximum level of the higher/softer state. The corresponding superluminal knot was fairly bright, but not as prominent as the other two. The spike in X-ray flux at 2005.7 coincides with a flare at $230 \mathrm{GHz}$, very close to the time of coincidence of the new superluminal knot and core at $43 \mathrm{GHz}$. This indicates that some of the $\mathrm{X}$-ray emission arises from the jet downstream of the central engine, which implies that the $\mathrm{X}$ ray dips in the coronal X-ray emission might be deeper than they appear in the RXTE light curve.

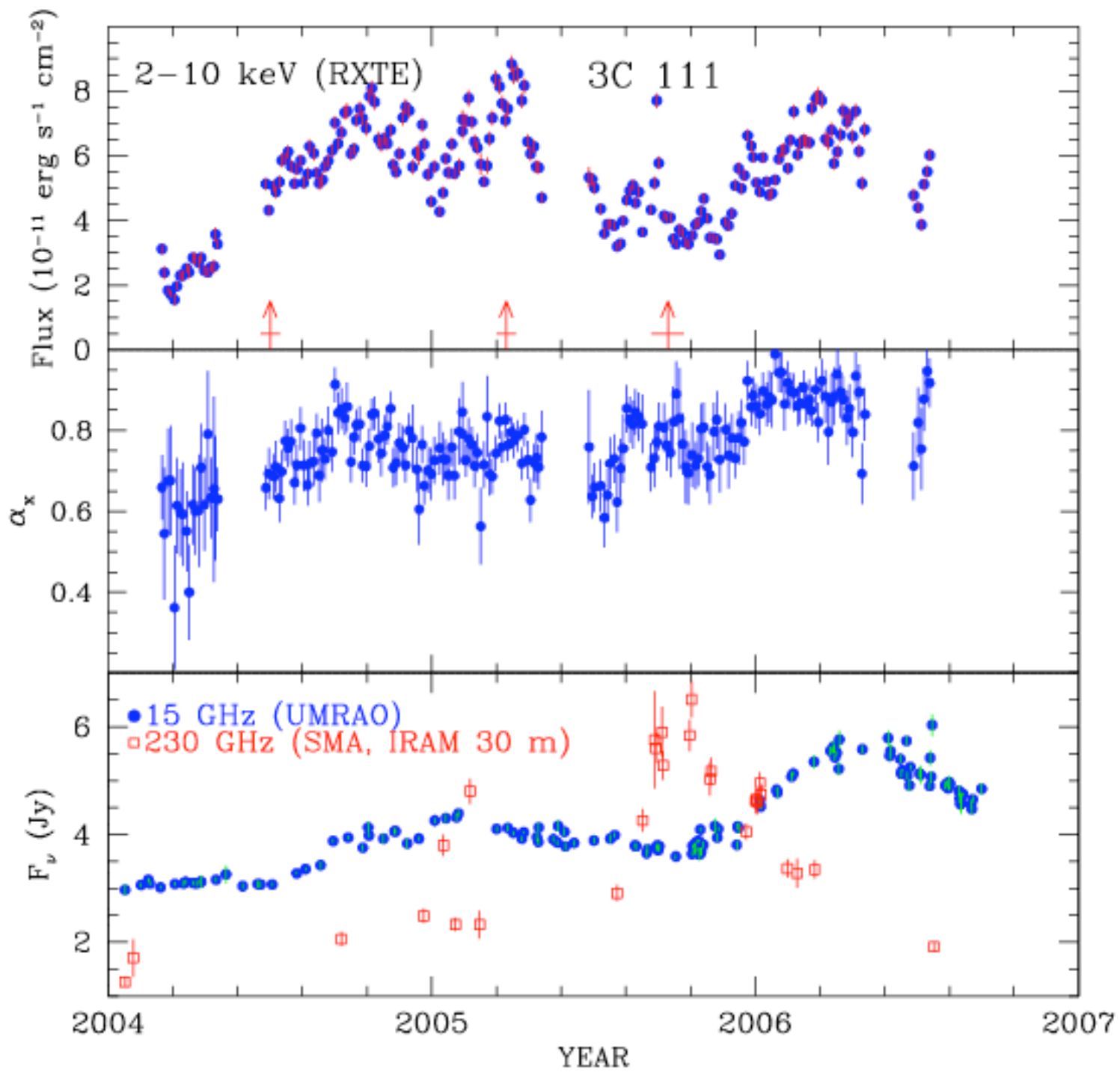

Figure 4. X-ray and radio light curves of the FR 2 radio galaxy 3C 111. Upward red arrows indicate times when superluminal knots were coincident with the core on $43 \mathrm{GHz}$ VLBA images (horizontal bars across the arrows show the uncertainty in this time). Every significant dip in X-ray flux and hardening of the X-ray spectrum precedes the ejection of a bright superluminal knot. 


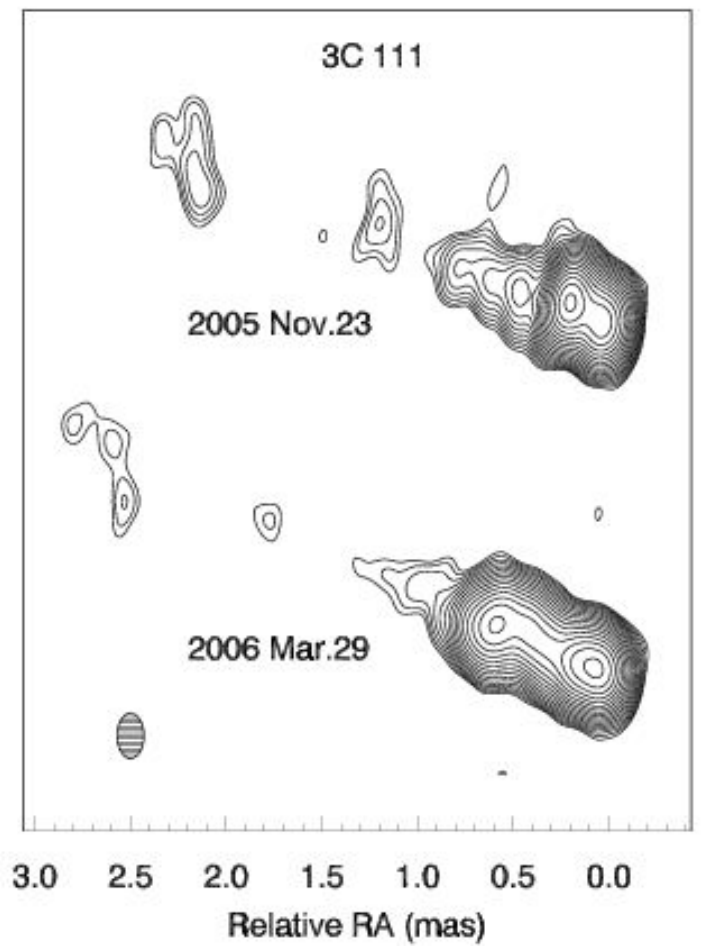

Figure 6. VLBA images at $43 \mathrm{GHz}$ of the radio galaxy $3 \mathrm{C} 111$ after the long low $\mathrm{X}$ ray state of 2005. A new superluminal knot moving at $4-5 c$, initially bright than the core, was trailed by an extended region of enhanced emission commensurate with the prolonged duration of the low X-ray state. This supports the conclusion that periods of low X-ray flux are associated with enhanced activity in the jet. Contours are in factors of $\sqrt{2}$ starting at $4.2 \mathrm{mJy} / \mathrm{beam}$, with peak at $1.7 \mathrm{Jy} /$ beam. The restoring beam is $0.24 \times 0.13$ millarcsec along position angle $-1^{\circ}$.

The physical cause of the connection between events in the central engine and the jet is currently a matter of considerable speculation. If the jet is magnetically launched, then there must be a link between the magnetic state at the base of the jet and the accretion state in the inner disk. One scenario, proposed by [25], involves a change in the magnetic field configuration in the inner disk from turbulent in the high-soft state to mainly poloidal in the low-hard state. The turbulence is needed for viscous heating, which in the XRB case leads directly to bright X-ray emission with a soft spectrum. In an AGN it causes strong ultraviolet emission, which is Compton scattered in the corona to a hard X-ray spectrum. If the field switches from chaotic to mainly poloidal - [25] suggest that this can occur by random nearalignment of the field in the relatively small number of turbulent cells in the inner disk - then the radiation in the inner disk will be quenched at the same time as energy flow into the jet is promoted. The transition back to the turbulent, radiative inner disk would need to be violent in order to send a shock wave (see [31]) down the jet. Perhaps global magnetic reconnection could cause this, but no detailed MHD model has been published to date.

Alternatively, it is possible that the "corona," where the X-ray emission seen in AGN supposedly arises from Compton upscattering of softer accretion-disk photons, might be the base of the jet or of a wind blown off the disk. If it is in fact the innermost section of the jet in the picture where the jet starts out relatively slow and speeds up with distance from the black hole, then the X-ray flux will be related to the number of electrons residing there. As mentioned above, the mass loading of the jet should affected the asymptotic Lorentz factor of the flow downstream. A decrease in the mass loading could then manifest itself as a decrease in scattered $\mathrm{X}$-ray emission near the disk (with the flatter-spectrum nonthermal X-ray emission from the downstream jet playing a larger relative role) and a time-delayed increase in the speed of the jet 
downstream. The latter will drive a shock wave, seen as a superluminal radio knot. It is difficult to say why the mass loading should change, since we do not understand the processes by which material from the disk and/or ergosphere are injected into the base of the jet. In fact, there is a centrifugal barrier that strongly inhibits particles from entering the jet (e.g., [29],[9]).

\section{Conclusions}

The relativistic jets from AGN can have bulk Lorentz factors $\Gamma$ ranging from close to one to at least 45. Most, however, fall in the lower end of this range. Acceleration up to the asymptotic flow speed probably occurs over an extended region, which explains why the collimation is tighter for higher values of $\Gamma$. The value of $\Gamma$ that a jet can reach depends on the ratio of magnetic to rest-mass energy density at the injection point. This links possible fluctuations in the mass loading to changes in the jet flow speed, which can cause shocks that appear as superluminal knots. Such a phenomenological model can explain the connection between X-ray dips and time-delayed appearances of superluminal knots in the radio jets of the radio galaxies $3 \mathrm{C} 120$ and $3 \mathrm{C} 111$. If this is the correct picture, the challenge remains to determine what controls the mass loading and how it relates to conditions in the disk.

\section{Acknowledgments}

The original work reported here was supported in part by National Science Foundation grant AST-0406865 and a number of NASA grants, most recently NNG05GM33G and NNG05GO46G. The VLBA is an instrument of the National Science Foundation operated by the National Radio Astronomy Observatory under cooperative agreement by Associated Universities Inc.

\section{References}

[1] S.D. Bloom et al., Radio, Millimeter-Submillimeter, and Infrared Spectra of Flat-Spectrum Extragalactic Radio Sources, AJ 198, 398-404 (1994).

[2] B.J. Burn, On the Depolarization of Discrete Radio Sources by Faraday Dispersion, MNRAS 133, 67-83 (1966).

[3] T.V. Cawthorne, Polarization of Synchrotron Radiation from Conical Shock Waves, MNRAS 367, 851-859 (2006).

[4] R.A. Daly \& A.P. Marscher, The Gas Dynamics of Compact Relativistic Jets, ApJ 334, 539-551 (1988).

[5] M. Eracleous, R. Sambruna, \& R.F. Mushotzky, Hard X-Ray Spectra of Broad-Line Radio Galaxies from the Rossi X-Ray Timing Explorer, ApJ 537, 654-666 (2000).

[6] R. Fender \& T. Belloni, GRS 1915+105 and the Disc-Jet Coupling in Accreting Black Hole Systems, Ann. Rev. Astr. Ap. 42, 317-364 (2004).

[7] J.L. Gómez et al., Hydrodynamical Models of Superluminal Sources, ApJ 482, L33-L36 (1997).

[8] J.L. Gómez, A.P. Marscher, A. Alberdi, S.G. Jorstad, \& C. García-Miró, Flashing Superluminal Components in the Radio Galaxy 3C 120, Science 289, 2317-2320 (2000). 
[9] J.F. Hawley \& J.H. Krolik, Magnetically Driven Jets in the Kerr Metric, ApJ 641, 103-116 (2006).

[10] D.A. Homan, et al., Intrinsic Brightness Temperature of AGN Jets, ApJ 642, L115-L118 (2006).

[11] P.A. Hughes, The Origin of Complex Behavior of Linearly Polarized Components in Parsec-Scale Jets, ApJ 621, 635-642 (2005).

[12] P.A. Hughes, H.D. Aller, \& M.F. Aller, Polarized Radio Outbursts in BL Lacertae II. The Flux and Polarization of a Piston-Driven Shock, ApJ 298, 301-315 (1985).

[13] P.A. Hughes \& L. Miller, Introduction: Synchrotron and Inverse-Compton Radiation, in Beams and Jets in Astrophysics, ed. P.A. Hughes (Cambridge U. Press), 1-51 (1991).

[14] C. Impey \& G. Neugebauer, Energy Distributions of Blazars, AJ 95, 307-351 (1988).

[15] S.G. Jorstad et al., Change in Speed and Direction of the Jet near the Core in the Quasar 3C 279, $A J$ 127, 3115-3120 (2004).

[16] S.G. Jorstad et al., Polarimetric Observations of 15 Active Galactic Nuclei at High Frequencies: Jet Kinematics from Bimonthly Monitoring with the Very Long Baseline Array, AJ 130, 1418-1466 (2005).

[17] S.G. Jorstad, et al. 2007, Polarimetric Observations of 15 Active Galactic Nuclei at High Frequencies: Correlated Polarization Behavior, AJ, submitted (2007).

[18] S.G. Jorstad \& A.P. Marscher, Highly Variable Apparent Speed in the Quasar 3C 279, Multiband Approach to AGN, ed. A.P. Lobanov \& T. Venturi, Mem. D. Soc. Astron. It. 76, 106-109 (2005).

[19] S.G. Jorstad, A.P. Marscher, J.R. Mattox, A.E. Wehrle, \& S.D. Bloom, Multi-Epoch Very Long Baseline Array Observations of EGRET-Detected Quasars and BL Lacertae Objects: Superluminal Motion of Gamma-Ray Bright Blazars, ApJS 134, 181-240 (2001).

[20] K.I. Kellermann et al., Sub-Milliarcsecond Imaging of Quasars and Active Galactic Nuclei. III. Kinematics of Parsec-scale Radio Jets, ApJ 609, 539-563 (2004).

[21] K.I. Kellermann \& I.I.K. Pauliny-Toth, The Spectra of Opaque Radio Sources, ApJ 155, L71-L78 (1969).

[22] A. Lähteenmäki \& E. Valtaoja, Inverse Compton Models for Active Galactic Nuclei Derived from Gamma-Ray and Radio Observations, ApJ 590, 95-108 (2003).

[23] A. Lähteenmäki, E., Valtaoja, \& K. Wiik, Total Flux Density Variations in Extragalactic Radio Sources. II. Determining the Limiting Brightness Temperature for Synchrotron Sources, ApJ 511, 112-117 (1999).

[24] M.L. Lister \& A.P. Marscher, Statistical Effects of Doppler Beaming and Malmquist Bias on FluxLimited Samples of Compact Radio Sources, ApJ 476, 572-588 (1997).

[25] M. Livio, J.E. Pringle, \& A.R. King, The Disk-Jet Connection in Microquasars and Active Galactic Nuclei, ApJ 593, 184-188 (2003).

[26] A.P. Marscher \& W.K. Gear, Models for High-Frequency Radio Outbursts in Extragalactic Sources with Application to the Early 1983 Millimeter to Infrared Flare of 3C273, ApJ 298, 114-127 (1985).

[27] A.P. Marscher, W.K. Gear, \& J.P. Travis, Variability of Nonthermal Continuum Emission in Blazars, in Variability of Blazars, ed. E. Valtaoja \& M. Valtonen (Cambridge U. Press), 85-101 (1992). 
[28] A.P. Marscher, et al. Observational Evidence for the Accretion-Disk Origin for a Radio Jet in an Active Galaxy, Nature 417, 625-627 (2002).

[29] J.C. McKinney, General Relativistic Magnetohydrodynamic Simulations of the Jet Formation and Large-Scale Propagation from Black Hole Accretion Systems, MNRAS 368, 1561-1582 (2006).

[30] D.L. Meier, S. Koide, \& Y. Uchida, Magnetohydrodynamic Production of Relativistic Jets, Science 291, 84-92 (2001).

[31] J.C.A. Miller-Jones et al., Multiple Relativistic Outbursts of GRS1915+105: Radio Emission and Internal Shocks, MNRAS 363, 867-881 (2005).

[32] I.F. Mirabel \& L.F. Rodríguez, Microquasars in Our Galaxy, Nature 392, 673-676 (1998).

[33] B.M. Peterson et al., Central Masses and Broad-Line Region Sizes of Active Galactic Nuclei. II. A Homogeneous Analysis of a Large Reverberation-Mapping Database, ApJ 613, 682-699 (2004).

[34] B.G. Piner, D. Bhatterai, P.G. Edwards, \& D.L. Jones, The Fastest Relativistic Jets: VLBA Observations of Blazars with Apparent Speeds Exceeding 25c, ApJ 640, 196-203 (2006).

[35] A.C.S. Readhead, Equipartition Brightness Temperature and the Inverse Compton Catastrophe, ApJ 426, 51-59 (1994).

[36] D.H. Roberts, R.I. Kollgaard, L.F. Brown, D.C. Gabuzda, \& J.F.C. Wardle, Milliarcsecond Polarization Structure of the Superluminal Quasar 3C 273, ApJ 360, 408-416 (1990).

[37] T. Savolainen, K. Wiik, E. Valtaoja, \& M. Tornikoski, Multifrequency VLBA Monitoring of $3 C 273$ during the INTEGRAL Campaign in 2003 - I. Kinematics of the Parsec Scale Jet from $43 \mathrm{GHz}$ Data, $A \& A$ 446, 71-85 (2006).

[38] N. Vlahakis \& A. Königl, A. Magnetic Driving of Relativistic Outflows in Active Galactic Nuclei. 1. Interpretation of Parsec-Scale Accelerations, ApJ 605, 656-661 (2004). 